\title{
Concussion
}

\section{The role of early intervention in improving the level of activities and participation in youths after mild traumatic brain injury: a scoping review}

\begin{abstract}
Mild traumatic brain injury in children can lead to persistent cognitive and physical symptoms which can have a negative impact on activities and participation in school and at play. Preventive treatment strategies are preferred because these symptoms are often not recognized and therefore not treated adequately. In this review clinical studies investigating interventions directed at pediatric mild traumatic brain injury are summarized, and clinical recommendations and directions for the future are provided. Results show that the literature is scarce and more high quality studies are needed. Information and education about the injury and its consequences are recommended, with additional follow-up consultation, including individualized advice and reassurance. The interventions should be family-centered and, ideally, the return to activity and participation should be graded and done step-by-step.
\end{abstract}

Keywords: activities $\bullet$ children $\bullet$ participation $\bullet$ traumatic brain injury

First draft submitted: 16 December 2016; Accepted for publication: 13 March 2017; Published online: 10 August 2017

Accidents can happen. Children and adolescents are often involved in accidents leading to traumatic brain injuries (TBI). The incidence of TBI in children between 0 and 18 years is $280-1373$ per 100,000 , but there is a large variation between studies and countries; most of these injuries are mild (mTBI) [1-8]. Most children recover completely after an mTBI, but $6-43 \%$ of children experience postconcussive symptoms (PCS) up to 6 months after the injury and beyond [9-12]. Persistent symptoms are found in the areas of physical, cognitive, emotional and behavioral functioning [13-16]. These consequences can lead to limitations in activities and participation such as returning to school and play [17-20]. Pediatric mTBI can also affect health-related quality of life (HRQOL) [21]. Children with PCS had significantly lower HRQOL scores at 4, 8 and 12 weeks postinjury than children without PCS and normal controls. Children without PCS had lower HRQOL scores than the norms at 4 and 8 weeks postinjury. School functioning scores were lower at all time points, regardless of the presence of PCS.

In general, children suffering the more severe forms of TBI (i.e., moderate and severe) are followed and receive rehabilitation treatment, but children with mTBI do not [22]. Both for professionals and for parents, the cognitive, emotional and behavioral problems are difficult to recognize and are therefore underestimated, underdiagnosed and not treated adequately [23]. Delayed recognition may, however, lead to unnecessary chronic and disruptive problems in activities and participation [13,24,25].

Several intervention strategies can be considered. First, all children and their parents can be given information and education on the possible consequences of an mTBI in order to prevent long-term problems. Second, children at risk of long-term problems can be identified at an early stage and information and education can be directed
Caroline van Heugten ${ }^{*, 1,2,3}$, Irene Renaud ${ }^{1,3,4}$ \& Christine Resch ${ }^{1,3}$

'Department of Neuropsychology \& Psychopharmacology, Faculty of Psychology \& Neuroscience, Maastricht University, Maastricht, The Netherlands ${ }^{2} \mathrm{~S}$ chool for Mental Health \& Neuroscience, Faculty of Health, Medicine \& Life Sciences, Maastricht University Medical Center, Maastricht, The Netherlands

${ }^{3}$ Limburg Brain Injury Center, Maastricht, The Netherlands

${ }^{4}$ Revant Rehabilitation Center, Breda, The Netherlands

*Author for correspondence: caroline.vanheugten@maastrichtuniversity.nl 
specifically toward this group. Early recognition and interventions are essential for children at risk of longterm problems [26,27]. It is, however, difficult to identify those at risk because the prognostic factors are not yet fully known. Third, information provision can be combined with routine follow-up aimed at detecting possible consequences. If disabling consequences are found, referral for treatment can be arranged. Finally, patients or parents who report consequences themselves, for instance to their general practitioner, can be referred for treatment. However, often this does not occur as these consequences are not recognized, either by the patients and parents themselves or by professionals. Accordingly, strategies for preventing long-term problems have been suggested to be the best treatment option [28,29].

In this paper we will review the available literature on early interventions for improving the level of activities and participation in children and adolescents with mTBI. On the basis of this overview we will formulate recommendations for clinical practice and suggest directions for future research.

\section{Review of the literature}

We did not perform a systematic review with a predefined search strategy because of the limited resources on this topic. Instead we performed a scoping review which uses a more broad research question: inclusion and exclusion criteria can be developed post hoc; study quality is not a priority; the review may or may not involve data extraction and offer a more qualitative than quantitative synthesis of evidence [30]. However, we did make some decisions concerning the inclusion of studies and we did extract data. To begin, we used the definition of mTBI as defined by the American Congress of Rehabilitation Medicine, that is: 'a Glasgow Coma Scale (GCS) score of 13-15 and at least one of the following: loss of consciousness of no more than $30 \mathrm{~min}$; Post Traumatic Amnesia no longer than $24 \mathrm{~h}$; any alteration in mental state at the time of the injury; focal neurological deficit(s) that may or may not be transient' [31]. We searched studies using the terms 'mild brain injury', 'mild traumatic brain injury', 'mild head injury' and 'concussion' in combination with 'children'/'childhood', 'youth', 'adolescents'/'adolescence', 'pediatric'/'paediatric' and 'interventions', 'activities' and participation'.

We selected clinical studies in which an intervention for children with mTBI was evaluated in the domains of activities and participation according to the framework of the International Classification of Functioning (ICF, WHO). Activities can be activities of daily life such as self-care, school, sports, hobby and play. Participation refers to the involvement of the children in life situations such as in domestic, community, social and civic life. We also considered family functioning or parent-child interaction as outcome domains. We did not include studies measuring outcome solely in terms of functioning such as motor functioning or cognitive functioning. We also did not include studies on interventions aiming at biochemical and neurochemical changes such as oxidative stress, inflammation and the neurometabolic cascade because these are mostly experimental and involve animal models, and outcome is measured mostly on the level of physiological and neurological functioning. Since pharmacological interventions are not primarily directed at improving the level of activities and participation, we excluded medication studies as well. If, however, medication was part of a more comprehensive program we did include the study.

Second, since the literature is still rather scarce in this area, we also considered studies in which children with mTBI were part of larger studies on moderate and severe pediatric TBI. Furthermore, studies that did not measure the level of activities and participation, but nevertheless investigated interventions for children with mTBI that might also be suitable for preventing problems with or improving the level of activities and participation, were included. Last, we discuss some potentially effective interventions from the literature on adult brain injury.

In addition to research papers, we considered reviews on interventions for children with (m)TBI [32-38] and searched for relevant references in these reviews. We extracted only studies in which interventions were evaluated. Papers describing treatment programs without an outcome evaluation were not considered; if these papers contained relevant recommendations for future research or clinical practice these are taken into account in our discussion section. If the review considered adults and children, we selected only the studies investigating children. If multiple papers were published about the same study, these are discussed separately only when they concern a different sample.

\section{Summary of the evidence on (early) interventions in pediatric $(m)$ TBI}

The following paragraphs describe the various identified types of intervention (e.g., information and education, online family problem-solving (FPS) interventions, cognitive and physical rest), separating interventions that are primarily aimed at all children with (m)TBI from interventions that target specific complaints in a subgroup of children who experience negative symptoms and/or are at risk of experiencing them in the future.

Table 1 provides an overview of studies investigating the effectiveness of interventions for activities and par- 
ticipation of children with mTBI. It also shows studies where children with $\mathrm{mTBI}$ were part of a larger group of children with moderate and/or severe TBI. Table 1 is the main table in which conclusions are drawn. The appendix displays studies of interventions for children with mTBI that are not directed at activities and participation, but nevertheless might be suitable for achieving improvement in these domains. These studies and studies on adults with mild forms of brain injury are described in the text only as 'additional information'.

It has to be noted that the definitions of TBI severity (i.e., mild, complicated mild, moderate or severe) were not consistent over the studies. Furthermore, the terms 'mild brain injury', 'mild traumatic brain injury', 'mild head injury' and 'concussion' may be used interchangeably [49,50]. The general clinical medical literature now uses mTBI [51]. The definitions used by the studies in this review vary. One study used the definition of the American Congress of Rehabilitation Medicine [40]. One study [31] made use of the Acute Concussion Evaluation form [52]. In two studies [53,54] the International Consensus on Concussion in Sport [55,56] was used. Other studies defined mTBI based on GCS scores (i.e., >12) and/or duration of loss of consciousness, duration of post-traumatic amnesia and presence/absence of focal neurological deficits [41-48,57,58]. For three studies $[39,59,60]$, the definition of mTBI was described as, that is, 'minor head injury', or 'diagnosed by a sports or rehabilitation medicine specialist'. Complicated mTBI was defined as a GCS score of greater than 12 with evidence of significant findings on clinical imaging [41-48,57]. Moderate TBI was defined as a GCS score of 9-12 [41-48]. Some studies combined complicated $\mathrm{mTBI}$ and moderate TBI and defined this group as moderate [41-48]. Severe TBI was defined as a GCS score of $<9[41-48]$.

\section{Information \& education}

Information about mTBI and education on signs and symptoms can be provided with the intention of improving the outcome of patients or their caregivers or both. Casey et al. were the first to study the effectiveness of an information and education protocol after childhood mTBI in reducing physical, social and/or behavioral problems postinjury [39]. Their intervention, consisting of a discharge interview during which the nurse explained a take-home booklet of symptoms that could be expected, instructions to follow at discharge and a follow-up telephone call $24 \mathrm{~h}$ after discharge, was found to be no more effective than the routine discharge sheet (i.e., a list of symptoms requiring reassessment at the hospital). However, in general, reporting of symptoms 1 month postinjury was low. A closer look at the data seemed to indicate that most symptoms at the 1-month follow-up occurred in children who had anxious parents, although this finding did not reach significance. Based on these findings, Casey et al. emphasize the importance of reassurance and education for parents about the signs and symptoms of minor head trauma (i.e., emphasizing that the symptoms are common and that they can be dealt with) [39]. This might aid children in returning to their daily activities and routines. Ponsford et al. developed just such an early education and reassurance intervention for children post-mTBI [40]. This study was the first to provide evidence that children who received a booklet describing symptoms and coping strategies within 1 week postinjury reported fewer PCS at 3 months postinjury, in comparison with those who did not receive this information. The intervention, however, had no direct effect on behavior in daily activities. However, the amount of difficulties that the study sample experienced in daily behavior before the interventions was already low. This low rate of symptoms might explain the lack of effect of the intervention in improving the functioning of children with mTBI.

Taken together, these studies seem to indicate that information and education interventions are useful in decreasing PCS in children with mTBI. These types of interventions could also be used to improve the level activities and participation of children with $\mathrm{mTBI}$ who report a decrease in or are at risk for problems in activities and participation (e.g., by preventing unnecessary absenteeism from school), but more research is necessary.

\section{Problem-solving interventions for families}

Four different but very similar interventions, two offline and two online, were identified in the literature for improving family and adolescent problem-solving skills following childhood TBI. The Counsellor Assisted Problem Solving (CAPS) intervention, the FPS intervention, the online FPS intervention, and the Teen Online Problem Solving (TOPS) intervention all provide therapistguided problem-solving training to adolescents with TBI and their families. In six to eight core sessions and, depending on the families' needs, up to four additional sessions, self-guided online learning of problem-solving skills, communication, self-management and self-regulation, as well as video-counseling with a therapist are offered. The (non-online) FPS intervention differs slightly, since the therapist and the families met at the families' homes or at the clinic for the therapy sessions, instead of participating in video-counseling. In most of the studies in the CAPS, (online) FPS or TOPS intervention, Internet Resource Comparison was used as a control intervention. Participants in the control group, if present, were provided with access to a website with 


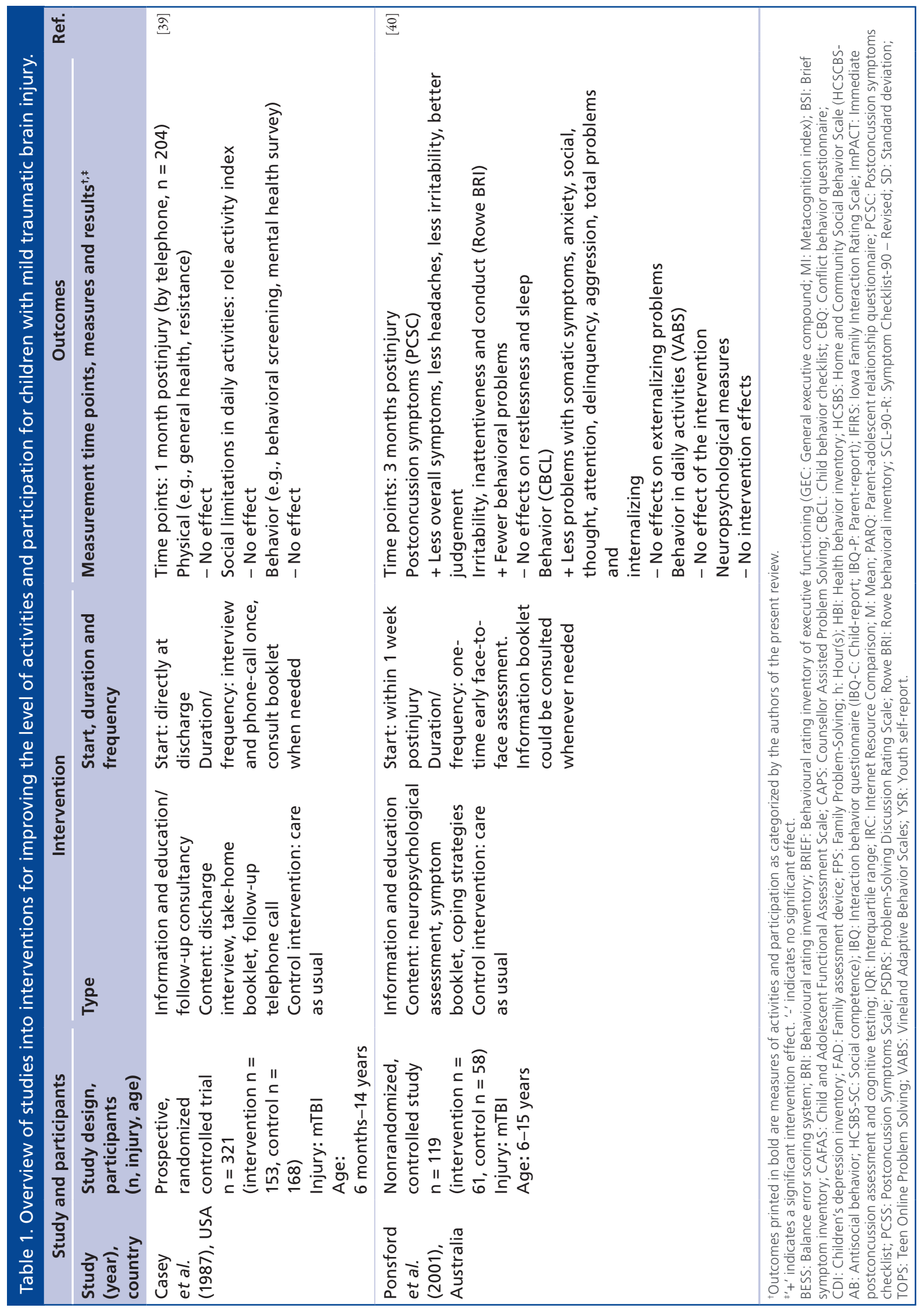




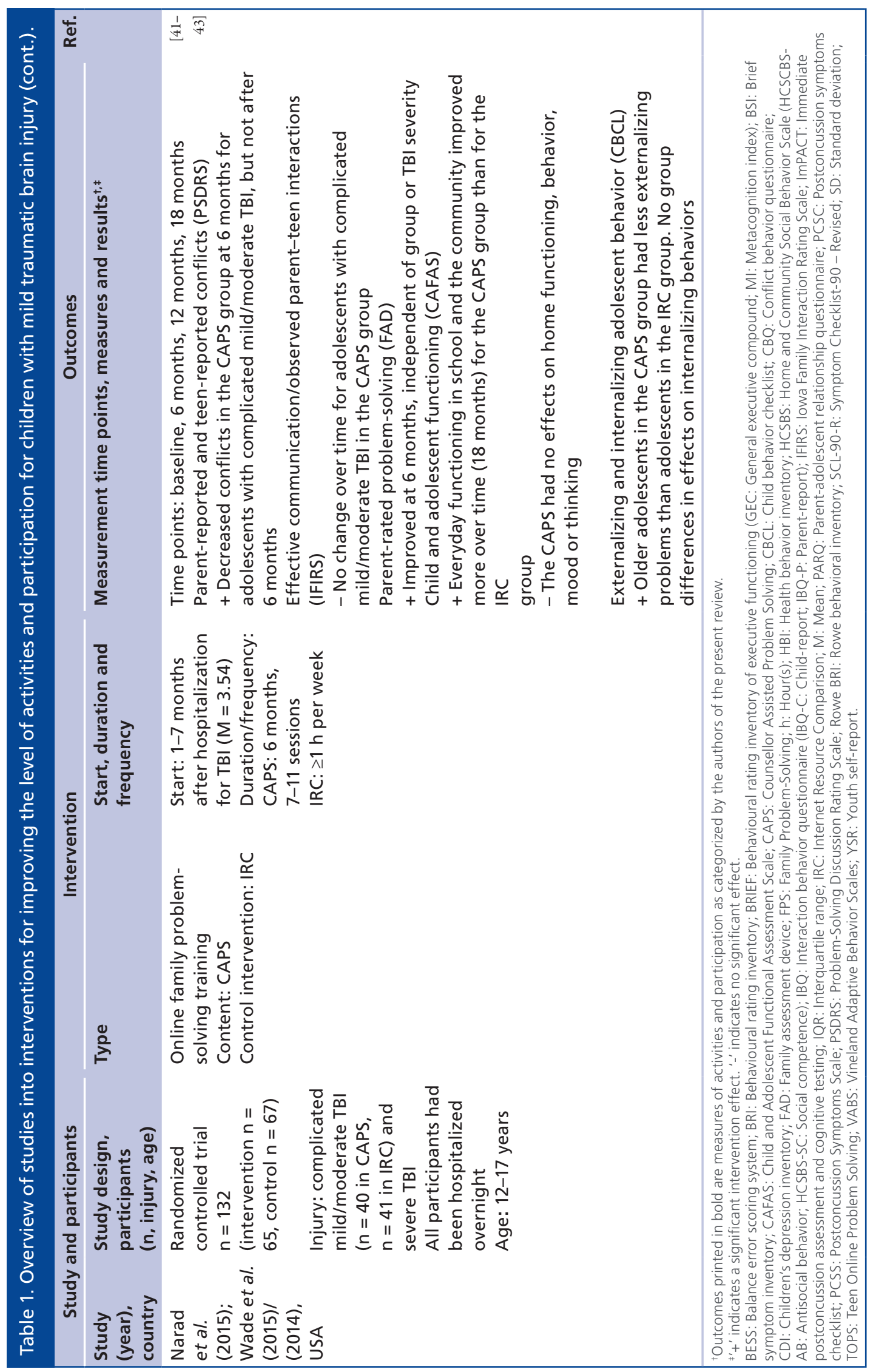




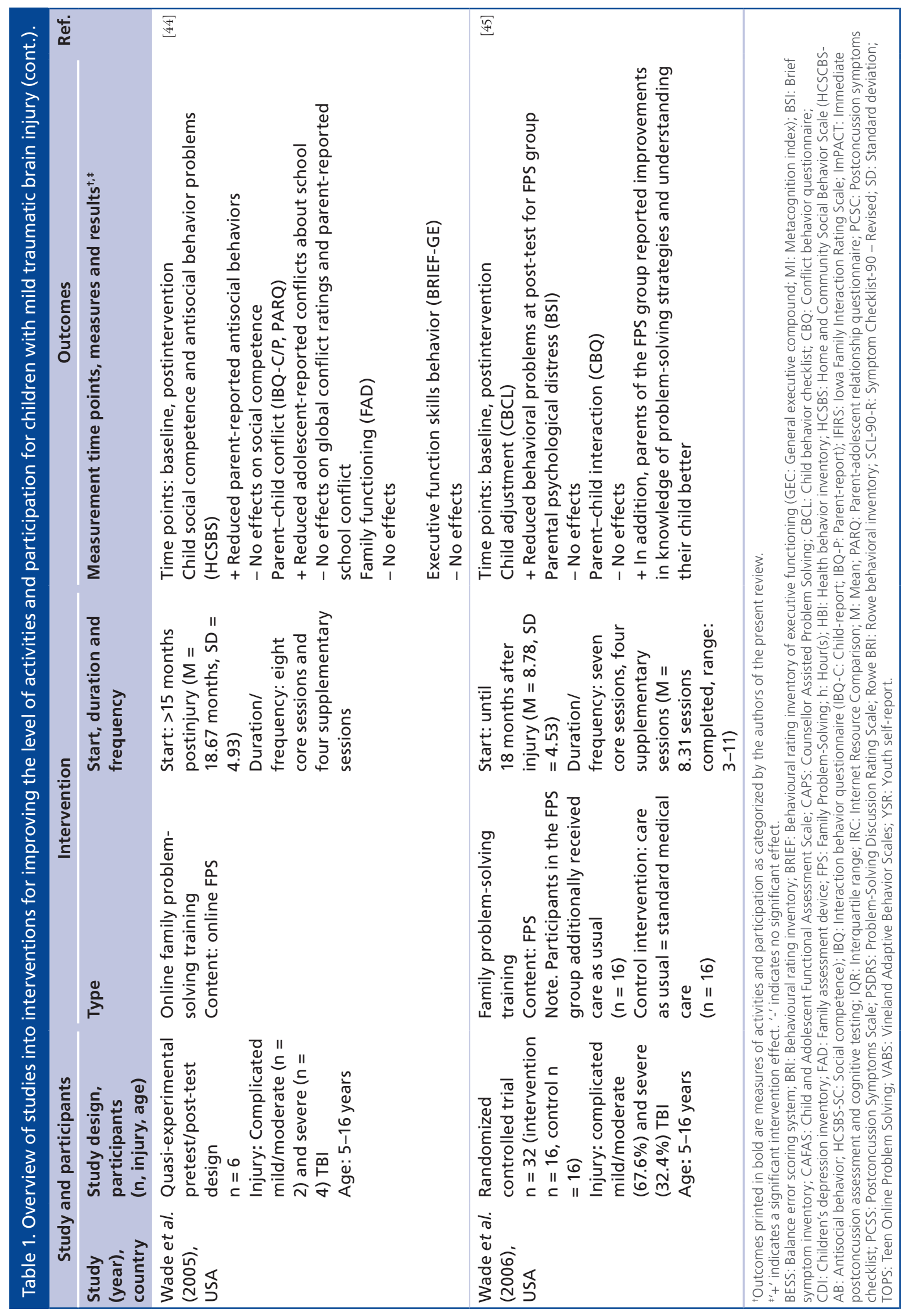




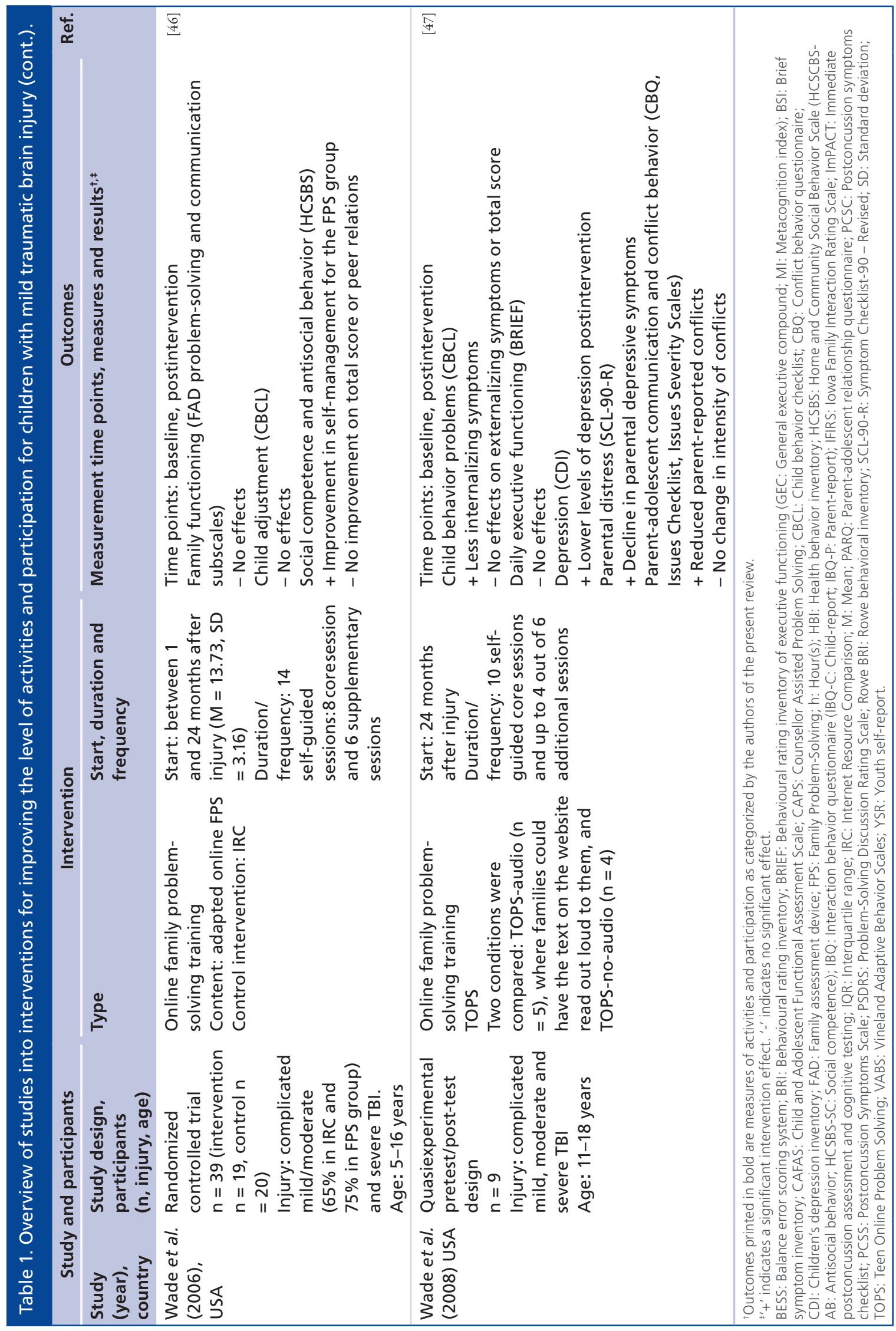




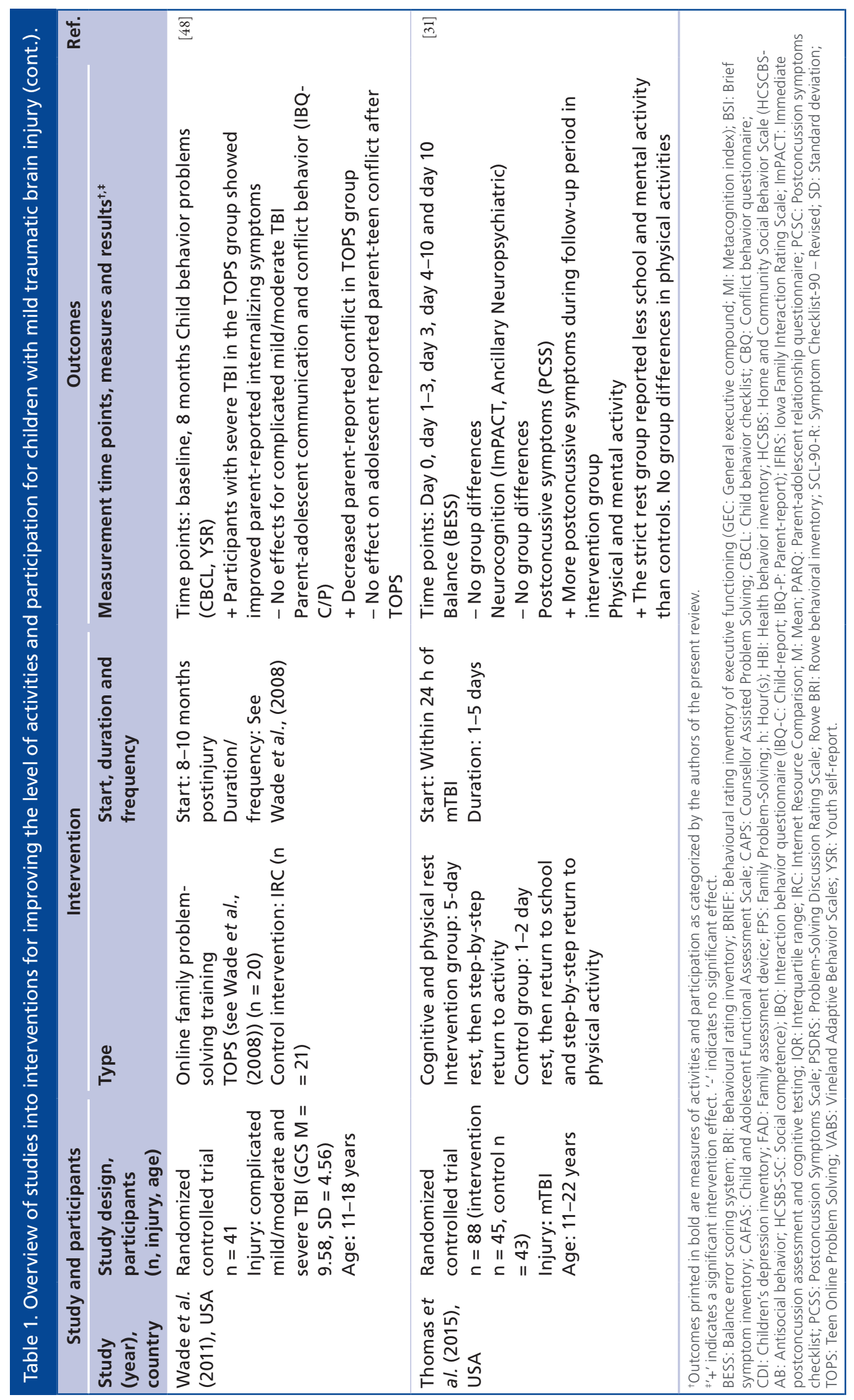


links to other websites about childhood brain injury and various brain-injury associations.

The different (online) family problem-solving interventions were investigated in six different studies, resulting in eight published articles. More specifically, one study investigated the CAPS [41-43], two studies examined the TOPS [47,48], one study investigated the non-online FPS [45], one study looked into an online version of the FPS [44] and one examined an adapted version of the FPS [46]. The design method of all of these studies varied (i.e., randomized controlled trials and quasi-experimental pretest/post-test experiments), and outcome measures varied as well (e.g., parent-child conflict is measured in three of the six studies, with two different measures). This makes it difficult to compare the interventions. Overall, the (online) family problemsolving interventions seem to have potential to improve child and family functioning, and therefore the level of activities and participation, of children with (m)TBI. More specifically, the CAPS intervention decreased parent- and teen-reported family conflict and improved everyday functioning in school and in the community of adolescents with complicated mild/moderate TBI. Communication and parent-teen interactions as well as home functioning, behavior, mood or thinking did not change with CAPS [41-43]. The TOPS intervention led to reduced parent-adolescent communication and conflict behavior and decreased parent-reported, but not adolescent-reported, conflict $[47,48]$. The online FPS intervention seems to be the least effective in improving the level of activities and participation of children with (m)TBI: no effects on parent-child interaction, global parent-child conflict or family functioning were found [45], although improvement was shown for adolescent-reported conflicts regarding school [44].

Several factors influencing effectiveness were identified in the studies investigating the CAPS, the (online) FPS and the TOPS interventions. For one, more improvement in child and adolescent functioning as well as in teen-reported family problem-solving skills and parent- and adolescent-reported child behavior after the (CAPS or adapted online FPS) intervention is related to lower parental education $[41,42,46]$. This seems to indicate that especially children with mTBI and lower-educated parents can benefit from a problemsolving intervention. Second, in contrast to younger adolescents, older adolescents showed positive behavioral changes and improvements in self-management after the CAPS and online FPS interventions $[41,43,46]$. Furthermore, the CAPS intervention was especially effective in improving school, work and community functioning, rather than other domains of functioning (e.g., home functioning, behavior and thinking). Last, parent-reported teen internalizing symptoms improved after the TOPS intervention, but only for participants with severe TBI. Taken together, these results indicate that factors such as parental education, age of the child, domain of functioning to be improved and severity of the injury can influence intervention effectiveness.

The effectiveness of the CAPS, the (online) FPS and the TOPS interventions was investigated in groups of children with complicated mild, moderate and severe TBI who were not selected based on their complaints and/or being at risk for these complaints. These interventions should, therefore, be categorized as interventions for the prevention of long-term symptoms. The effectiveness of these interventions in a more selected group of children with TBI remains unknown. Furthermore, since children with complicated mTBI were always analyzed together with children with moderate TBI, it remains unclear what effect these interventions would have on the level of activities and participation and other outcomes in a group solely of children with (complicated) mTBI.

\section{Cognitive \& physical rest}

Rest during the acute stage of recovery, reduction of physical and cognitive activities, monitoring symptoms in collaboration with their parents, taking rest breaks after returning to school, spending fewer hours at school, being allowed more time to take exams, having help with schoolwork, gradually returning to sports and reducing time spent with the computer, reading and writing are among the recommendations for managing symptoms after mTBI in children [54-56,59,61]. Cognitive and physical rest recommendation is often part of the care as usual for children with mTBI and is also described in protocols such as the return-to-learn and the return-to-play protocols [62]. However, in reviewing the literature, we encountered only one study investigating the effects of cognitive and physical rest on the level of activities and participation for children with mTBI [31]. To determine if strict cognitive and physical rest was beneficial with regard to postinjury recovery, patients were divided into two groups: one group was recommended to have 1-2 days of rest, while the other group was advised to have strict rest for 5 days. Both groups were recommended to return to activity step by step after the days of rest. Results showed that strict rest caused children with mTBI to report more PCS. Furthermore, in comparison with children who had only 1-2 days of rest, the more rested children experienced a decrease in the level of activities and participation. This is not surprising, since per definition cognitive and physical rest entails restricted level of activities and participation. The effects of cognitive and physical rest on the level of activities and participation over the long term still have to be determined. 


\section{Additional information} Information \& education

Kirkwood et al. performed a pilot study investigating a one-time neuropsychological consultation consisting of interviews with parents and children and a standardized battery of tests [57]. Feedback on the results was provided to the families by a neuropsychologist, including general education about concussion, information about injury and noninjury-related factors contributing to the child's specific symptoms and recommendations for addressing any concerns. They found that PCS decreased significantly following the consultation. Unlike the two studies reported above, the study by Kirkwood et al. was focused on children with mTBI who were already reporting problems for some time [57]. The finding that the intervention was effective in decreasing PCS in these children is promising, indicating that interventions consisting of information and/or education are suited not only for the prevention of symptoms but also for more specific treatment.

\section{Follow-up consultancies}

In a study by Bell et al. follow-up contact by telephone was found to be effective in reducing symptoms after mTBI [63]. This study, however, was performed with children aged 16 years and older and with adults after mTBI and the effect on younger children is unknown (and therefore not in Table 1). Furthermore, the effect on the level of activities and participation was not measured.

\section{Cognitive \& physical rest}

From the studies we reviewed for the present article, no consensus can be derived regarding the benefits of cognitive and physical rest for children with mTBI. One study supports rest as an effective form of care after mTBI in children. Independent of when a minimum of 1 week of cognitive and physical rest was described (i.e., 1-7 days, 8-30 days or more than 31 days postinjury), PCS were reduced and cognitive functioning was improved [54]. Another study found no association between the prescription of cognitive rest and the duration of symptoms [53]. While these results seem contradictory, methodological differences between the studies have to be taken into account when interpreting the results. While the first study [54], finding benefits of cognitive and physical rest, examined only the presence of PCS at one time point, the other study [53], failing to find an association between rest and PCS, investigated the duration of symptoms over time. Furthermore, while the first study examined a period of cognitive and physical rest of approximately 1 week, the duration of rest used in the second study is not clear. This should be taken into account, since, as described above, increasing the duration of cognitive and physical rest from 1-2 days to 5 days was found to have negative effects for children with mTBI [31]. The relation between the duration of PCS and the duration of cognitive and physical rest needs further research.

\section{Combined interventions}

Some interventions in children with mTBI are comprised of a combination of components. For example, Gagnon et al. used graded guided rehabilitation as their primary intervention [59]. The intervention stops when children are symptom-free. Children who do not remain symptom-free receive a return appointment for re-evaluation, education and a weekly follow-up. This combination continues until the child remains symptom-free. The results of this study suggest that involvement in controlled and closely monitored rehabilitation in the postacute period may promote recovery in children and adolescents who present with slow recovery after mTBI.

Another combined intervention consisted of education and advice on avoiding analgesic overuse, avoiding any opiate medications and encouraging light exercise when PCS persisted for 3 months or longer postinjury [58]. Furthermore, prophylactic medications were selected based on comorbidities by a neurologist with expertise in acquired brain injury and headache disorders. A marked reduction in the frequency of headaches was reported in half of the cases after the intervention, while $45 \%$ reported complete resolution of headaches.

A combined collaborative care intervention, consisting of care management, CBT and possible psychopharmacological consultation, was examined by McCarty et al. [60]. They found that efforts to systematically implement collaborative care treatment approaches for slow-to-recover adolescents may be useful given the reductions in postconcussive and co-occurring psychological symptoms in addition to improved quality of life.

All of the above-mentioned combined interventions were conducted with children and adolescents who experienced symptoms after mTBI. Although the results are promising, the influence of such interventions on preventing symptoms in the first place was not studied, nor was the influence on activities and participation.

\section{Evidence from literature on mild forms of brain injury in adults}

Interventions designed to reduce symptoms after mTBI in adults have been investigated by several researchers. Providing information with educational brochures or sessions about common symptoms after mTBI, including reassurance of recovery, the likely time course of recovery and information on how to cope with symptoms are among the intervention strategies [64-67], as 
are neuropsychological assessments and follow-up contact by telephone.

More specifically, for adults with mTBI, Paniak et al. [68] showed that an education-oriented single session and a more extensive assessment, education and treatment-as-needed intervention showed similar results on symptom-related, functional and vocational variables 3-4 months after the initial assessment. These results were maintained at the 12-month followup, while most improvements in both groups were seen in the first 3 months [65]. Recently similar results were found in a study where a high-risk mTBI group received a doctor's visit in addition to written information, in comparison with a control group receiving only written information [69]. The high-risk group was defined as patients having three or more PCS at 10 days postinjury. The groups did not differ in terms of symptoms, anxiety or depression at the 3-month follow-up. Ponsford et al. [66] studied the effectiveness of an extra follow-up moment in which an information booklet on mTBI was given to adults 1 week after visiting the emergency department. The information booklet contained information about $\mathrm{mTBI}$, the possible consequences and time course and coping strategies to deal with these consequences. In comparison with a control group receiving no information booklet, the patients in the intervention reported significantly fewer symptoms and were less stressed at the 3-month follow-up. Nygren-de Boussard et al. [70] conducted a systematic review on the evidence of nonsurgical interventions for persistent symptoms after mTBI and also showed the beneficial effects of early, reassuring educational interventions.

Based on the effectiveness of these education interventions, Moulaert et al. [71] developed an early neurologically focused intervention for patients with hypoxic brain injury due to a cardiac arrest. Cardiac arrest can lead to hypoxic brain injury which can be comparable to the diffuse damage seen in mTBI. The intervention consists of screening for cognitive and emotional problems, provision of information and support, promotion of selfmanagement strategies and referral to further specialized care if indicated. This intervention was found to be feasible in clinical practice [72] and both clinically effective [73] and cost-effective [74] in comparison with care as usual. Patients in the intervention group had a better quality of life, a better overall emotional state and fewer symptoms of anxiety 1 year postcardiac arrest. Moreover, more people returned to work 3 months postinjury.

Nelson, Sheese and Hammeke propose treatment strategies both on the basis of clinical consensus and the limited evidence base [75]. In addition to education about mTBI, possible persistent symptoms and the natural course of recovery, and reassurance of a good outcome, they suggest reducing activity levels and refraining from hazardous behaviors during the acute phase and a gradual return to lifestyle activities as symptoms permit. Professionals should carefully monitor and offer early intervention for adverse emotional responses, offer symptom-specific treatment when needed, and enable ready access to providers during the first weeks of recovery. Al Sayegh, Sandford and Carson also suggest that information, education and reassurance alone may not be sufficient in reducing PCS [76]. They suggest that cognitive behavioral therapy with psychotherapeutic elements or mindfulness or relaxation techniques may lead to increased improvements. These interventions are directed mainly toward the reduction of PCS and to a lesser extent toward improving the level of activities and participation.

\section{Conclusion}

Most interventions for children with mTBI are designed to reduce symptoms, such as headaches, cognitive problems or other PCS, and are not specifically designed to improve the level of activities and participation. Interventions consisting of information and education seem effective in preventing symptoms when reassurance is combined with information. Reassurance can be optimized by including a follow-up consultation by telephone, during which individual concerns can be addressed. Family problem-solving interventions are effective in improving child and family functioning but have not been investigated for a group of children with only mTBI. There is no consensus on the benefits of cognitive and/or physical rest, but graded activity procedures seem effective in supporting return to school, sports and play. Combined interventions including medication have not been offered as a preventive strategy and the effects of such interventions on the level of activities and participation are unknown.

In addition to studies investigating the effectiveness of interventions for children with mTBI, several protocols and recommendations have been published on returning to activity and returning to school, for which no studies have been made regarding their effectiveness. Protocols on returning to activity can be divided into graded, or step-by-step protocols, and severity-oriented guidelines on how to build levels of activity. The protocols with a graded approach reported the following six steps: no activity; light aerobic exercise; sport-specific exercise; noncontact training drills; full contact practice; and return to play [77-79]. Guidelines that are severity-oriented focus on the severity of symptoms or the numbers of previous concussions. For example, when a child's first concussion is considered to be mild, the guideline would recommend returning to play after being symptom-free for 1 week. For a concussion that is 
considered severe, the child should be symptom free for a month [80]. Furthermore, severity-oriented guidelines focused on injury-related factors such as confusion, loss of consciousness and post-traumatic amnesia [81,82]. A protocol on returning to school was provided by Master $e t$ al. [62]. Their step-by-step protocol consisted of the following steps: no activity; gradual reintroduction of cognitive activity; homework at home before schoolwork at school; school re-entry; gradual reintegration into school; and full return to school and cognitive workload. Sady et al. recommend a graduated accommodation-based education plan with similar steps [83]. Furthermore, several other recommendations on returning to school can be found in the literature, such as monitoring and support, removal of distractions, excuse or absence from class or activity, and increased time to complete tests and tasks [84]. Unfortunately, most of these protocols focused on sports concussions in school athletes and the effects of these protocols were not examined. Studies on interventions that are set up in order to directly prevent long-term restrictions on participation in activities for children and adolescents after mTBI are, to our knowledge, unknown.

In conclusion, evidence suggests that information and education should always be offered, ideally followed by a consultation in which personalized reassurance is given. The family should be involved and problem-solving interventions seem effective. In addition, clinical recommendations suggest a step-by-step return to cognitive and physical activities, not only restricted to sports.

\section{Future perspective}

This review shows that the literature on early interventions to improve the level of activities and participation in is scarce with regard to pediatric mTBI. There are not many high-quality studies available and the comparability of studies is limited because of variation in population (i.e., separate studies on $\mathrm{mTBI}$ ), definitions (i.e., the definition of TBI), the aim of the intervention (i.e., prevention or treatment) and outcome domains (i.e., symptoms or activities and participation) and out- come measures. Ideally the first step should be to identify children at risk of long-term problems by conducting longitudinal prospective cohort studies, followed by high-quality randomized controlled trials in which targeted interventions are investigated. Given the current economic pressures in healthcare, these evaluation studies should include analyses of both clinical effectiveness and cost-effectiveness, and consider potential implementation in clinical practice at an early stage. Research studies investigating preventive strategies are challenging because of recruitment (i.e., can we detect all cases), selection bias (i.e., will all cases participate or only those having complaints or fearing consequences) and follow-up (i.e., will all cases remain in the study).

Currently we are conducting a randomized controlled trial in which the early intervention Brains ahead! is being evaluated in terms of effectiveness on participation in activities in comparison with standard care [85]. The Brains ahead! intervention is a combination of screening for mTBI symptoms, psychoeducation and follow-up. Outcome is measured 3 and 6 months postinjury. The primary outcome measure is the Child and Adolescent Scale of Participation [86]; in addition, other measures of activities, participation, quality of life and child behavior are performed. We hope to have recruited 140 children by the end of 2017 .

\section{Financial \& competing interests disclosure}

The authors have no relevant affiliations or financial involvement with any organization or entity with a financial interest in or financial conflict with the subject matter or materials discussed in the manuscript. This includes employment, consultancies, honoraria, stock ownership or options, expert testimony, grants or patents received or pending, or royalties. No writing assistance was utilized in the production of this manuscript.

\section{Open access}

This work is licensed under the Creative Commons Attribution 4.0 License. To view a copy of this license, visit http://creativecommons.org/licenses/by/4.0/

\section{Executive summary}

- Pediatric mild traumatic brain injury may lead to reduced activities and participation in a considerable number of victims.

- Intervention strategies to prevent long-term problems are preferable to treatment of long-term problems.

- Interventions should include information and education on the injury and its possible consequences and include follow-up consultations aimed at reassurance.

- Interventions should be family-centered.

- Step-by-step return to activities is recommended.

- High-quality randomized studies are necessary.

- Consensus on definitions and outcome domains and measurements increases comparability and, therefore, enlarges the evidence base. 


\section{References}

1 Hawley CA, Ward AB, Long J, Owen DW, Magnay AR. Prevalence of traumatic brain injury amongst children admitted to hospital in one health district: a populationbased study. Injury 34(4), 256-260 (2003).

2 Kim HB, Kim DK, Kwak YH et al. Epidemiology of traumatic head injury in Korean children. J. Korean Med. Sci. 27(4), 437-442 (2012).

3 Koepsell TD, Rivara FP, Vavilala MS et al. Incidence and descriptive epidemiologic features of traumatic brain injury in King County, Washington. Pediatrics 128(5), 946-954 (2011).

4 Langlois JA, Rutland-Brown W, Thomas KE. The incidence of traumatic brain injury among children in the United States: differences by race. J. Head Trauma Rehabil. 20(3), 229-238 (2005).

5 Mckinlay A, Grace R, Horwood L, Fergusson D, Ridder EM, Macfarlane M. Prevalence of traumatic brain injury among children, adolescents and young adults: prospective evidence from a birth cohort. Brain Inj. 22(2), 175-181 (2008).

6 Schneier AJ, Shields BJ, Hostetler SG, Xiang H, Smith GA. Incidence of pediatric traumatic brain injury and associated hospital resource utilization in the United States. Pediatrics 118(2), 483-492 (2006).

7 Styrke J, Stålnacke B-M, Sojka P, Björnstig U. Traumatic brain injuries in a well-defined population: epidemiological aspects and severity. J. Neurotrauma 24(9), 1425-1436 (2007).

8 Wu X, Hu J, Zhuo L et al. Epidemiology of traumatic brain injury in eastern China, 2004: a prospective large case study. J. Trauma Acute Care Surg. 64(5), 1313-1319 (2008).

9 Barlow KM, Crawford S, Stevenson A, Sandhu SS, Belanger F, Dewey D. Epidemiology of postconcussion syndrome in pediatric mild traumatic brain injury. Pediatrics 126(2), e374-e381 (2010).

10 Dahl E, Von Wendt L, Emanuelson I. A prospective, population-based, follow-up study of mild traumatic brain injury in children. Injury 37(5), 402-409 (2006).

11 Hawley CA, Ward AB, Magnay AR, Long J. Outcomes following childhood head injury: a population study. J. Neurol. Neurosurg. Psychiatry 75(5), 737-742 (2004).

12 Ruff R. Two decades of advances in understanding of mild traumatic brain injury. J. Head Trauma Rehabil. 20(1), 5-18 (2005).

13 Blinman TA, Houseknecht E, Snyder C, Wiebe DJ, Nance ML. Postconcussive symptoms in hospitalized pediatric patients after mild traumatic brain injury. J. Pediatr. Surg. 44(6), 1223-1228 (2009).

14 Hawley C, Ward AB, Magnay AR, Mychalkiw W. Return to school after brain injury. Arch. Dis. Child. 89(2), 136-142 (2004).

15 Hung R, Carroll LJ, Cancelliere C et al. Systematic review of the clinical course, natural history, and prognosis for pediatric mild traumatic brain injury: results of the International Collaboration on Mild Traumatic Brain Injury Prognosis. Arch. Phys. Med. Rehabil. 95(3), S174-S191 (2014).
16 Taylor HG, Orchinik LJ, Minich N et al. Symptoms of persistent behavior problems in children with mild traumatic brain injury. J. Head Trauma Rehabil. 30(5), 302-310 (2015).

17 Bedell GM, Dumas HM. Social participation of children and youth with acquired brain injuries discharged from inpatient rehabilitation: A follow-up study. Brain Inj. 18(1), 65-82 (2004).

18 De Kloet AJ, Gijzen R, Braga LW, Meesters JJ, Schoones JW, Vliet Vlieland TP. Determinants of participation of youth with acquired brain injury: a systematic review. Brain Inj. 29(10), 1135-1145 (2015).

19 Foo WS, Galvin J, Olsen J. Participation of children with $\mathrm{ABI}$ and the relationship with discharge functional status. Dev. Neurorehabil. 15(1), 1-12 (2012).

20 Van Tol E, Gorter JW, Dematteo C, Meester-Delver A. Participation outcomes for children with acquired brain injury: a narrative review. Brain Inj. 25(13-14), 1279-1287 (2011).

21 Novak Z, Aglipay M, Barrowman N et al. Association of persistent postconcussion symptoms with pediatric quality of life. JAMA Pediatr. 170(12), e162900 (2016).

22 Van Pelt ED, De Kloet A, Hilberink SR et al. The incidence of traumatic brain injury in young people in the catchment area of the University Hospital Rotterdam, The Netherlands. Eur. J. Paediatr. Neurol. 15(6), 519-526 (2011).

23 Levin HS, Hanten G, Roberson G et al. Prediction of cognitive sequelae based on abnormal computed tomography findings in children following mild traumatic brain injury. J. Neurosurg. Pediatr. 1(6), 461-470 (2008).

24 Anderson V, Catroppa C. Advances in postacute rehabilitation after childhood-acquired brain injury: a focus on cognitive, behavioral, and social domains. Am. J. Phys. Med. Rehabil. 85(9), 767-778 (2006).

25 Königs M, Heij HA, Van Der Sluijs JA et al. Pediatric traumatic brain injury and attention deficit. Pediatrics 136(3), 534-541 (2015).

26 Anderson VA, Catroppa C, Haritou F, Morse S, Rosenfeld JV. Identifying factors contributing to child and family outcome 30 months after traumatic brain injury in children. J. Neurol. Neurosurg. Psychiatry 76(3), 401-408 (2005).

27 Zemek RL, Farion KJ, Sampson M, Mcgahern C. Prognosticators of persistent symptoms following pediatric concussion: a systematic review. JAMA Pediatr. 167(3), 259-265 (2013).

28 Redelmeier DA, Raza S. Concussions and repercussions. PLoS Med. 13(8), e1002104 (2016).

29 Sariaslan A, Sharp DJ, D’onofrio BM, Larsson H, Fazel S. Long-term outcomes associated with traumatic brain injury in childhood and adolescence: a nationwide Swedish cohort study of a wide range of medical and social outcomes. PLoS Med. 13(8), e1002103 (2016).

30 Armstrong R, Hall BJ, Doyle J, Waters E. 'Scoping the scope' of a cochrane review. J. Public Health 33(1), 147-150 (2011).

31 Thomas DG, Apps JN, Hoffmann RG, Mccrea M, Hammeke T. Benefits of strict rest after acute concussion: a randomized controlled trial. Pediatrics 135(2), 213-223 (2015). 
32 Borg J, Holm L, Peloso P et al. Non-surgical intervention and cost for mild traumatic brain injury: results of the WHO Collaborating Centre Task Force on Mild Traumatic Brain Injury. J. Rehabil. Med. 36(0), 76-83 (2004).

33 Caskey RC, Nance ML. Management of pediatric mild traumatic brain injury. Adv. Pediatr. 61(1), 271-286 (2014).

34 Gravel J, D'angelo A, Carrière B et al. Interventions provided in the acute phase for mild traumatic brain injury: a systematic review. Syst. Rev. 2(1), 1 (2013).

35 Kirkwood MW, Yeates KO, Taylor HG, Randolph C, Mccrea M, Anderson VA. Management of pediatric mild traumatic brain injury: a neuropsychological review from injury through recovery. Clin. Neuropsychol. 22(5), 769-800 (2008).

36 Rose SC, Weber KD, Collen JB, Heyer GL. The diagnosis and management of concussion in children and adolescents. Pediatr. Neurol. 53(2), 108-118 (2015).

37 Ross KA, Dorris L, Mcmillan T. A systematic review of psychological interventions to alleviate cognitive and psychosocial problems in children with acquired brain injury. Dev. Med. Child Neurol. 53(8), 692-701 (2011).

38 Winkler R, Taylor NF. Do children and adolescents with mild traumatic brain injury and persistent symptoms benefit from treatment? A systematic review. J. Head Trauma Rehabil. 30(5), 324-333 (2015).

39 Casey R, Ludwig S, Mccormick MC. Minor head trauma in children: an intervention to decrease functional morbidity. Pediatrics 80 (2), 159-164 (1987).

40 Ponsford J, Willmott C, Rothwell A et al. Impact of early intervention on outcome after mild traumatic brain injury in children. Pediatrics 108(6), 1297-1303 (2001).

41 Narad ME, Minich N, Taylor HG et al. Effects of a webbased intervention on family functioning following pediatric traumatic brain injury. J. Dev. Behav. Pediatr. 36(9), 700-707 (2015).

42 Wade SL, Kurowski BG, Kirkwood MW et al. Online problem-solving therapy after traumatic brain injury: a randomized controlled trial. Pediatrics 135(2), e487-e495 (2015).

43 Wade SL, Stancin T, Kirkwood M, Brown TM. Counselorassisted problem solving (CAPS) improves behavioral outcomes in older adolescents with complicated mild to severe TBI. J. Head Trauma Rehabil. 29(3), 198 (2014).

44 Wade SL, Wolfe CR, Brown TM, Pestian JP. Can a webbased family problem-solving intervention work for children with traumatic brain injury? Rehabil. Psychol. 50(4), 337 (2005).

45 Wade SL, Michaud L, Brown TM. Putting the pieces together: preliminary efficacy of a family problem-solving intervention for children with traumatic brain injury. J. Head Trauma Rehabil. 21(1), 57-67 (2006).

46 Wade SL, Carey J, Wolfe CR. The efficacy of an online cognitive-behavioral family intervention in improving child behavior and social competence following pediatric brain injury. Rehabil. Psychol. 51(3), 179 (2006).

47 Wade SL, Walz NC, Carey JC, Williams KM. Preliminary efficacy of a web-based family problem-solving treatment program for adolescents with traumatic brain injury. J. Head Trauma Rehabil. 23(6), 369-377 (2008).

48 Wade SL, Walz NC, Carey J et al. Effect on behavior problems of teen online problem-solving for adolescent traumatic brain injury. Pediatrics 128(4), e947-e953 (2011).

49 Petchprapai N, Winkelman C. Mild traumatic brain injury: determinants and subsequent quality of life. A review of the literature. J. Neurosci. Nurs. 39(5), 260-272 (2007).

50 Prevention NCFI, Control. Report to congress on mild traumatic brain injury in the United States: steps to prevent a serious public health problem. Centers for Disease Control and Prevention (2003). www.cdc.gov/traumaticbraininjury/pdf/mtbireport-a.pdf

51 Earth J, Varney RN, Ruchinskas RA, Francis JP. Mild head injury: the new frontier in sports medicine. In: The Evaluation and Treatment of Mild Traumatic Brain Injury (1st Edition). Psychology Press, NJ, USA, 81-98 (1999).

52 Gioia GA, Collins M, Isquith PK. Improving identification and diagnosis of mild traumatic brain injury with evidence: psychometric support for the acute concussion evaluation. J. Head Trauma Rehabil. 23(4), 230-242 (2008).

53 Gibson S, Nigrovic LE, O'brien M, Meehan Iii WP. The effect of recommending cognitive rest on recovery from sport-related concussion. Brain Inj. 27(7-8), 839-842 (2013).

54 Moser RS, Glatts C, Schatz P. Efficacy of immediate and delayed cognitive and physical rest for treatment of sportsrelated concussion. J. Pediatr. 161(5), 922-926 (2012).

55 Mccrory P, Meeuwisse W, Johnston K et al. Consensus statement on concussion in sport: the 3rd International Conference on Concussion in Sport held in Zurich, November 2008. Br. J. Sports Med. 43(Suppl. 1), i76-i84 (2009).

56 Mccrory P, Meeuwisse WH, Aubry M et al. Consensus statement on concussion in sport: the 4th International Conference on Concussion in Sport held in Zurich, November 2012. Br. J. Sports Med. 47(5), 250-258 (2013).

57 Kirkwood MW, Peterson RL, Connery AK, Baker DA, Forster J. A pilot study investigating neuropsychological consultation as an intervention for persistent postconcussive symptoms in a pediatric sample. J. Pediatr. 169, 244. e241-249.e241 (2016).

58 Kuczynski A, Crawford S, Bodell L, Dewey D, Barlow KM. Characteristics of post-traumatic headaches in children following mild traumatic brain injury and their response to treatment: a prospective cohort. Dev. Med. Child Neurol. 55(7), 636-641 (2013).

59 Gagnon I, Galli C, Friedman D, Grilli L, Iverson GL. Active rehabilitation for children who are slow to recover following sport-related concussion. Brain Inj. 23(12), 956-964 (2009).

60 Mccarty CA, Zatzick D, Stein E, Wang J, Hilt R, Rivara FP. Collaborative care for adolescents with persistent postconcussive symptoms: a randomized trial. Pediatrics 138(4), e20160459 (2016).

61 Grady MF, Master CL, Gioia GA. Concussion pathophysiology: rationale for physical and cognitive rest. Pediatr. Ann. 41(9), 377-382 (2012). 
62 Master CL, Gioia GA, Leddy JJ, Grady MF. Importance of 'return-to-learn' in pediatric and adolescent concussion. Pediatr. Ann. 41(9), e180-e185 (2012).

Bell KR, Hoffman JM, Temkin NR et al. The effect of telephone counselling on reducing post-traumatic symptoms after mild traumatic brain injury: a randomised trial. J. Neurol. Neurosurg. Psychiatry 79(11), 1275-1281 (2008).

64 Mittenberg W, Tremont G, Zielinski RE, Fichera S, Rayls KR. Cognitive-behavioral prevention of postconcussion syndrome. Arch. Clin. Neuropsychol. 11(2), 139-145 (1996).

65 Paniak C, Toller-Lobe G, Reynolds S, Melnyk A, Nagy J. A randomized trial of two treatments for mild traumatic brain injury: 1 year follow-up. Brain Inj. 14(3), 219-226 (2000).

66 Ponsford J, Willmott C, Rothwell A et al. Impact of early intervention on outcome following mild head injury in adults. J. Neurol. Neurosurg. Psychiatry 73(3), 330-332 (2002).

67 Wade D, King N, Wenden F, Crawford S, Caldwell F. Routine follow-up after head injury: a second randomised controlled trial. J. Neurol. Neurosurg. Psychiatry 65(2), 177-183 (1998).

68 Paniak C, Toller-Lobe G, Durand A, Nagy J. A randomized trial of two treatments for mild traumatic brain injury. Brain Inj. 12(12), 1011-1023 (1998).

69 Matuseviciene G, Borg J, Stålnacke B-M, Ulfarsson T, De Boussard C. Early intervention for patients at risk for persisting disability after mild traumatic brain injury: a randomized, controlled study. Brain Inj. 27(3), 318-324 (2013).

70 Nygren-De Boussard C, Holm LW, Cancelliere C et al. Nonsurgical interventions after mild traumatic brain injury: a systematic review. Results of the International Collaboration on Mild Traumatic Brain Injury Prognosis. Arch. Phys. Med. Rehabil. 95(3), S257-S264 (2014).

71 Moulaert VR, Verbunt JA, Bakx WG et al. 'Stand still..., and move on', a new early intervention service for cardiac arrest survivors and their caregivers: rationale and description of the intervention. Clin. Rehabil. 25(10), 867-879 (2011).

72 Moulaert VR, Van Haastregt JC, Wade DT, Van Heugten CM, Verbunt JA. 'Stand still..., and move on', an early neurologically-focused follow-up for cardiac arrest survivors and their caregivers: a process evaluation. BMC Health Serv. Res. 14(1), 1 (2014).

73 Moulaert VR, Van Heugten CM, Winkens B et al. Early neurologically-focused follow-up after cardiac arrest improves quality of life at one year: a randomised controlled trial. Int. J. Cardiol. 193, 8-16 (2015).
74 Moulaert VR, Goossens M, Heijnders IL, Verbunt JA, Van Heugten CM. Early neurologically focused follow-up after cardiac arrest is cost-effective: a trial-based economic evaluation. Resuscitation 106, 30-36 (2016).

75 Nelson Sheese A, Hammeke T. Rehabilitation from postconcussion syndrome: nonpharmacological treatment. In: Concussion. Karger Publishers, Basel, 149-160 (2014).

76 Al Sayegh A, Sandford D, Carson AJ. Psychological approaches to treatment of postconcussion syndrome: a systematic review. J. Neurol. Neurosurg. Psychiatry 81(10), 1128-1134 (2010).

77 Halstead ME, Walter KD. Sport-related concussion in children and adolescents. Pediatrics 126(3), 597-615 (2010).

78 Johnston K, Barootes B, Barwitzki G et al. Guidelines for assessment and management of sport-related concussion (reprinted). Clin. J. Sport Med. 10(3), 209-211 (2000).

79 Kissick J. New Concussion Management Guidelines: Concussion Question and Answer Document for Physicians. www.mosessport.com

80 Cantu RC. Posttraumatic retrograde and anterograde amnesia: pathophysiology and implications in grading and safe return to play. J. Athl. Train. 36(3), 244 (2001).

81 Quality Standards Subcommittee of the American Academy of Neurology. Practice parameter: the management of concussion in sports (summary statement). Neurology 48(3), 581-585 (1997).

82 Colorado Medical Society. Report of the sports medicine committee: guidelines for the management of concussion in sports. JAMA 266(20), 2867-2869 (1991).

83 Sady MD, Vaughan CG, Gioia GA. School and the concussed youth: recommendations for concussion education and management. Phys. Med. Rehabil. Clin. N. Am. 22(4), 701-719 (2011).

84 Mcgrath N. Supporting the student-athlete's return to the classroom after a sport-related concussion. J. Athl. Train. 45(5), 492-498 (2010).

85 Renaud I, Lambregts SA, De Kloet A, Catsman-Berrevoets CE, Van De Port I, Van Heugten CM. Activities and participation of children and adolescents after mild traumatic brain injury and the effectiveness of an early intervention: the Brains Ahead! study design. Trials 17(1), 236 (2016).

86 Bedell G. Further validation of the Child and Adolescent Scale of Participation (CASP). Dev. Neurorehabil. 12(5), 342-351 (2009). 